\title{
Big impact, small effort Implementing a low-cost, highly inclusive recognition program with huge results
}

Iike many organizations, the Wayne - State University Libraries have historically struggled with a recognition system that had the potential to acknowledge people from all areas of the libraries, especially in a way that everyone felt like part of the process. Over the years, we tried things like recognition toolkits for managers, a dedicated recognition team, and formal awards ceremonies, but none were wholly embraced, and they quickly fizzled out as interest waned. Shrinking budgets added to the challenge, as funding for awards became harder and harder to obtain.

Though we hadn't yet found anything that worked, it didn't mean that people weren't interested in recognition. It was regularly addressed in library leadership meetings and commented on by staff. Staff and administration were clamoring for something we could start doing immediately.

People voiced that they wanted something they could do quickly and easily. They wanted something that could be submitted through a web form and accessible from a computer, phone, or tablet. It had to be fast and easy so as not to become a cumbersome task for our already busy staff. They wanted staff at all levels to be able to receive or give recognition for even seemingly small things-day-today things that people do that make them great colleagues and create extraordinary experiences for our users. Though highend awards are great, many of our staff found them to be exclusionary and set up to recognize only "major" achievements. They felt the recognition should be publicly shared. Someone remarked "I wish it was as easy as just giving a high five in front of everyone!" As it turns out, it was.

We created an electronic form that tied all submissions to our organizational values. After you nominated someone, you had to check off the values that they demonstrated before you could submit. We chose unique prizes that we didn't typically give-gift cards to local businesses instead of university swag. It was very important to us that the staff view this as something completely new and different-from the structure to the prize choices. Within two weeks, High Five was born. It was released as a pilot to see if it would catch on, and we were thrilled to see that it immediately took off.

We initially sent out a general call to staff announcing the program and en-

Jill Wurm is associate director of marketing, communications, and organizational development at Wayne State University Library System, email:ae0831@ wayne.edu

(C) 2018 Jill Wurm 
couraged everyone who was involved in creating it to promote it in meetings and to other staff. The first message sent out read:

Got a hard-working, outgoing, allaround excellent co-worker? Give them a High Five! Every employee is eligible; no good deed is too small. Plus, your colleague will be entered into a quarterly drawing for one of three \$20 gift cards from local businesses. Your High Five will be shared via the ALLWSULS list, so everyone in the Libraries can see just what makes your colleague so remarkable. And you'll get the satisfaction of knowing that excellence has been justly rewarded. Up High, people! High Fiving is fast and easy-just follow the link or click the button below to fill out a short form. High Five is open to all library employees so please share this link with your students so they can also participate and be sure to bookmark it yourself! https://library.wayne.edu/highfive/."

When someone submits the form, a copy of that submission is automatically sent to the program coordinator. From there it is vetted for anything that might require further clarification or follow up. Next, we send an email to the person or team nominated to let them know they've been High Fived and that this will be shared with the staff in a weekly digest. Some people prefer not to be publicly recognized so this gives them the opportunity to opt out of the announcement.

What are people recognizing? Absolutely everything. Things like covering hours or reference shifts are popular, but it's just as common to see someone recognized for a good deed, like stepping in to help with a difficult user, assistance in planning an event, or going out of his or her way to rearrange a schedule to make a meeting. Half of the fun is the writing style of the nominator: while some go straight to the
High Five, others spin well-crafted stories that not only provide recognition to the nominee but huge entertainment value to the readers.

Another big bonus is that people are continually reminded of our library values, resulting in an increased awareness of demonstrating them.

We compile all the High Fives for the week into a single email that is sent out every Friday and add all the names to a master list in Excel. Names are added as many times as someone is nominated. For example, if someone received three nominations that week, his or her name goes in three times. Before the quarterly drawings these are merged into label-sized slips of paper and the dean selects the winners.

High Five has been in place for just over a year and is still going strong, averaging between three-to-eight High Fives each week. In the last year alone, more than 300 staff members were recognized. We held four prize drawings, several as part of allstaff meetings and one as a silent drawing (hand-picked by the dean).

To keep the program from feeling stale and predictable, in the summer of 2017 we debuted a monthly drawing, where a single winner is chosen to receive specially branded High Five swag. In this case, a choice of a stainless steel water bottle or stainless steel travel mug. Since that first monthly drawing, we've seen an uptick in participation, with several people remarking that the rewards seem more attainable with the addition of the monthly drawings.

With the program in full swing, the only official reminders we send out are the emails that acknowledge that week's staff being recognized (which encourage people to continue High Fiving and provide a link to the form), but High Five has increasingly been a topic of conversation between colleagues at meetings and even around the water coolers. It isn't rare to hear "Oh, I have to remember to submit a High Five to thank him (or her) for that." 
At the one-year mark, we sent out a survey to find out how people were feeling about High Five now. Were they still High Fiving? Why or why not? What could we do to get them High Fiving more or to make it more inclusive?

With a $20 \%$ response rate to the survey, the responses told us that people were still enjoying the program and felt empowered
"It is a great way of facilitating peer-topeer recognition."

"I like being able to recognize people who may not normally have a chance to be recognized."

"I've learned about people that I didn't know before."

High Five has been an easy program

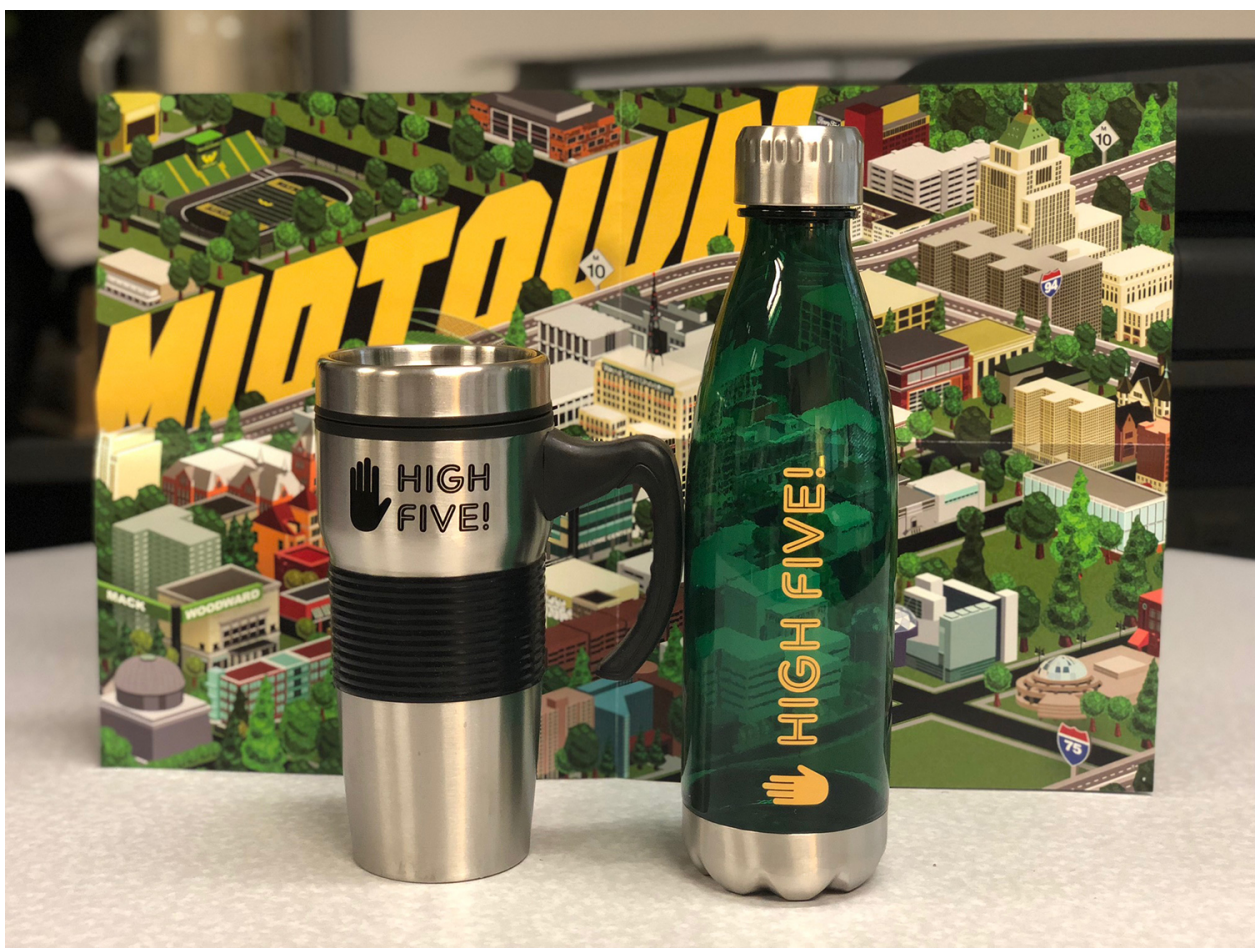

\section{Examples of High Five program swag.}

by High Five. Seventy-three percent of those who responded had High Fived someone else, and $70 \%$ had been High Fived themselves.

Every level of employee had participated in the last year, library and academic staff, managers, directors, and even the dean. The vast majority of the responses were excited about the opportunity to recognize others and find out things about colleagues that they may not know as well:

"Feels good and is easy to do."

"It is encouragement for everyone." to implement but has yielded big results. Staff are engaged and thinking about recognition in a way that they haven't before in our libraries.

High Fiving is becoming part of our organizational culture. However, this peerto-peer program isn't one that can only uniquely work here. It could work well in any organization that is looking for a fast, easy, cost-efficient way to get an informal recognition program in place.

For the Wayne State University Libraries, this very simple program has helped cultivate a culture of recognition that we hope to grow in years to come. $z$ 